Proceedings of SALT 24: 640-655, 2014

\title{
The sticky reading: VP ellipsis without parallel binding*
}

\author{
Patrick D. Elliott \\ University College London
}

\author{
Andreea C. Nicolae \\ ZAS Berlin
}

\author{
Yasutada Sudo \\ University College London
}

\begin{abstract}
VP Ellipsis (VPE) whose antecedent VP contains a pronoun famously gives rise to an ambiguity between strict and sloppy readings. Since Sag's (1976) seminal work, it is generally assumed that the strict reading involves free pronouns in both the elided VP and its antecedent, whereas the sloppy reading involves bound pronouns. The majority of current approaches to VPE are tailored to derive this parallel binding requirement, ruling out mixed readings where one of the VPs involves a bound pronoun and the other a free pronoun in parallel positions. Contrary to this assumption, it is observed that there are cases of VPE where the antecedent VP contains a bound pronoun but the elided VP contains a free E-type pronoun anchored to the quantifier, in violation of parallel binding. We dub this the 'sticky reading' of VPE. To account for it, we propose a new identity condition on VPE which is less stringent than is standardly assumed. We formalize this using an extension of Roberts's (2012) Question under Discussion (QuD) theory of information structure.
\end{abstract}

Keywords: VP ellipsis, strict/sloppy identity, pronominal binding, focus, Question under Discussion

\section{Introduction}

It has been famously observed by Sag (1976) that pronouns give rise to an ambiguity in a VP Ellipsis (VPE) context. An example such as (1) is claimed to be ambiguous between so-called strict and sloppy readings.

(1) Jonny totaled his car, and Kevin did $\langle\ldots\rangle$ too.

Under the sloppy reading, probably the most salient here, Jonny totaled Jonny's car, and Kevin totaled Kevin's car. Under the strict reading, Jonny totaled Jonny's car,

* We would like to thank Klaus Abels, Annabel Cormack, Ezra Keshet, Matthew Reeve, Gary Thoms, and the audience at the poster session of SALT 24 for useful discussion and comments. All remaining errors are our own. 
The sticky reading

and Kevin also totaled Jonny's car. ${ }^{1}$ According to Sag's (1976) classic analysis, the strict and sloppy readings correspond to having either a free or a bound pronoun respectively in the ellipsis site (e-site). The overt pronoun may either co-refer with the referring expression Patrick, or it may be bound by it, but it is subject to the following requirement, which we dub parallel binding.

\section{Parallel Binding}

a. If the e-site contains a free pronoun, the corresponding pronoun in the antecedent VP must be free;

b. If the e-site contains a bound pronoun, the corresponding pronoun in the antecedent VP must be bound.

Sag's (1976) parallel binding has been highly influential, and most, perhaps all, theories of VPE found in the current literature that we are familiar with are tailored to enforce it in one way or another (e.g., Dalrymple, Shieber \& Pereira 1991; Rooth 1992; Fiengo \& May 1994; Heim \& Kratzer 1998; Büring 2005). Contrary to this long-held assumption, however, we present data showing that there are instances of VPE that do not involve parallel binding, which we dub the 'sticky reading'. By way of illustration, consider (3).

(3) None of the authors proofread his paper. So the editor did $\langle\ldots\rangle$ (instead).

In addition to the strict and sloppy readings, (3) has a third reading that can be paraphrased as 'The editor proofread all the authors' papers'. We will claim that this reading, which we dub the sticky reading, involves a definite term in the e-site in the following manner. ${ }^{2}$

(4) $\quad$ None of the authors $]^{x}$ proofread his $x$ paper. So the editor did /proof read them/the authors' papers $\rangle$ (instead).

The definite term here could be a pronoun or a definite description. We remain agnostic as to which is contained in the VPEs in our examples, leaving it as a possibility that both options are in principle available. But for ease of exposition, we sometimes speak as if it is a definite description.

Importantly, the sticky reading violates parallel binding in that the pronoun in the antecedent VP is bound by the quantifier, while the elided VP has no bound pronoun in the corresponding position. The purpose of the present paper is to discuss the consequences of this observation for the theory of VPE. In particular, we argue

1 There is also a third, strict reading on which the referent of the overt pronoun is resolved as some contextually-salient individual, e.g. Daniel. Under this reading, Jonny totaled Daniel's car, and Kevin also totaled Daniel's car.

2 Throughout the paper, binding and anaphoric relations are indicated by superscripts and subscripts, and the e-site is marked by \langle\rangle . 
that the sticky reading is problematic by zooming in on Rooth's (1992) Parallelism Condition as a representative theory. Instead of the Parallelism Condition, we put forward two conditions on VPE. Although our account is still preliminary, it successfully captures examples of sticky readings like (3) in simple cases, unlike previous theories.

This paper is structured as follows. In Section 2, we introduce crucial data illustrating the availability of the sticky reading. In Section 3, we argue that the sticky reading poses a serious challenge for theories that require parallel binding, focusing on the version of the problem for Rooth 1992. Then in Section 4 we will propose two conditions on VPE, the Focus Condition and the Discourse Condition. The latter is stated in terms of an extension of Roberts's (2012) QuD framework. Section 5 concludes.

\section{Sticky readings}

We are actually not the first to point out the existence of sticky readings. For instance, Fiengo \& May (1994: 101) discuss the following example.

(5) Many students think they are smart, but the professor doesn't $\langle\ldots\rangle$.

Citing Lappin (1992) and T. Nishigauchi (p.c. to Fiengo \& May (1994)) the authors note that despite the fact that the pronoun in the antecedent clause is interpreted as a bound variable, the pronoun in the ellipsis site is interpreted as an E-type pronoun denoting the students who consider themselves to be smart. This is a sticky reading in our sense.

Likewise, Dalrymple, Shieber \& Pereira (1991: 429) present the following data.

(6) Every student revised his paper, and then Bill did $\langle\ldots\rangle$.

This sentence has a reading where Bill revised all of the students' papers. Again, despite the fact that the pronoun his in the antecedent clause is bound, the e-site does not seem to involve a bound pronoun, contradicting parallel binding.

Nonetheless, these authors do not see the above examples as particularly problematic. Fiengo \& May (1994) maintain that in both clauses the pronouns are anaphoric to the quantificational NP many students (in their parlance marked as $\alpha$-occurrences). Thus, according to their view, the sticky reading of (5) is a sub-case of the strict reading. While we will argue for a very similar view where the e-site contains a definite description dependent on the quantifier, Fiengo \& May's analysis runs into problems. Whereas their analysis is applicable to the above example, (5), cases like (3) and (6), where the pronoun in the antecedent is clearly a bound pronoun (or what they call a $\beta$-occurrence), do not seem to be amenable to the same explanation. 
The sticky reading

Dalrymple et al. (1991), on the other hand, suggest that in (6) the quantificational phrase every student takes scope over both sentences, thus binding both the pronoun in the antecedent VP and the pronoun in the e-site, as in the following paraphrase:

(7) Every student revised his paper and then Bill revised it.

(Dalrymple et al. 1991: 429)

This is an instance of so-called 'telescoping', i.e., a phenomenon where certain quantifiers (apparently) extend their scope across sentential boundaries, binding pronouns in a subsequent sentence (Poesio \& Zucchi 1992). Here are some examples of telescoping that are independent of the sticky reading.

a. Each degree candidate accepted his diploma and his mother took a picture.

b. Each degree candidate walked to the stage. He took his diploma from the Dean and returned to his seat.

(Keshet 2008: 324)

Dalrymple et al.'s idea is, therefore, that examples like (6) generally involve telescoping, whereby the sticky reading can be thought of as a sub-case of the sloppy reading. However, there are examples of sticky readings that are not amenable to this analysis, but can only be correctly analysed if we take the VPE to contain a definite term. We present six such cases here.

First, we observe that negative quantifiers also give rise to sticky readings, as we already saw in (3), repeated here.

(9) None of the authors proofread his paper. So the editor $\operatorname{did}\langle\ldots\rangle$.

The second sentence has a sticky reading that says that the editor proofread all of the authors' papers. Notice especially that this entails that the editor did proofread at least one paper. If we analyse this example with telescoping, however, the resulting reading, as illustrated informally in (10), will be too weak.

$\neg \exists x[x$ is one of the authors and $x$ proofread $x$ 's paper.

So the editor proofread $x$ 's paper]

In particular, (10) does not entail that the editor did any proofreading. On the other hand, if the VPE contains a definite term, as in (11), the correct truth-conditions are derived.

(11) So the editor did 〈proofread the authors' papers $\rangle$.

Secondly, the telescoping analysis makes the wrong predictions about the scope interaction between the quantifier and the connective in sentences like the following.

(12) Each of the boys revised his paper. So Prof. Jones didn't. 
This example has a distinct reading where Prof. Jones didn't do any revising because every one of the boys has revised his paper. In order words, the universal quantifier takes scope below the connective so. If the quantifier took scope over both sentences, this scopal relation would remain unaccounted for. On the other hand, under our analysis, the correct scope relation is captured, with so referring back to the first sentence as a whole as the reason for the second sentence.

Thirdly, the sticky reading is observed with a universal quantifier embedded in a finite clause, which is considered to be a scope island.

(13) John told me that every boy revised his paper. In that case, Prof. Jones mustn't have $\langle\ldots\rangle$.

The second sentence here can be understood as: 'In that case, Prof. Jones mustn't have revised the boys' papers'. This is unexpected if telescoping is what is behind the sticky reading, while it is exactly what our analysis with a definite term would predict.

Fourthly, several of our informants found a continuation with a singular definite degraded.

a. ?* Each of the boys revised his paper. So Prof. Jones didn't revise [his paper]/it.

b. ?* None of the boys revised his paper. So Prof. Jones revised his paper/it.

The very same speakers who find the continuation with a singular pronoun degraded (including one of the authors), nonetheless find VPE under the sticky reading perfectly acceptable. This suggests that for these speakers at least, the sticky reading cannot be explained by invoking an exceptionally wide-scope quantifier. On the other hand, these judgments are accounted for under our analysis with a definite description with a reasonable assumption that telescoping is subject to various pragmatic constraints that are violated in (14) for some speakers (see below), while E-type anaphora with a plural definite term is perfectly felicitous for all speakers in these examples.

Fifthly, if the subject of the second sentence is plural, as in the following example, a cumulative interpretation is possible under the sticky reading.

(15) None of the boys revised his paper. So three professors did $\langle\ldots\rangle$ instead.

The second sentence here has a reading where the three professors each revised a subset of the papers written by the boys, but collectively covers all their papers. Again, telescoping cannot account for this interpretation, while our analysis with a definite term predicts it, given that the following sentence has a cumulative reading (see, e.g. Beck \& Sauerland 2000 for detailed discussion of the cumulative reading).

(16) Three professors revised the boys' papers. 
The sticky reading

Finally, Keshet (2008) observes that telescoping is sensitive to the coherence relations (in the sense of Kehler 2002, 2011) that hold between sentences. For instance, a telescoping reading isn't available if the two sentences stand in Kehler's EXPLANATION coherence relation, as in the following example, where the first sentence is the explanandum, and the second the explanans.

$$
\text { \# Each of my friends is dishonest. Because he's a politician. }
$$

On the other hand, the sticky reading is in principle available when the two sentences stand in an EXPLANATION relation, as demonstrated by the following example, which can express that Prof. Jones revised the students' papers.

(18) Each of the students didn't revise his paper. Because Prof. Jones did $\langle\ldots\rangle$.

This observation suggests that constraints on the availability of the sticky reading aren't reducible to constraints on telescoping. Under our analysis with a definite term, on the other hand, this observation is unproblematic, as the following discourse is perfectly felicitous.

(19) Each of the students didn't revise his paper. Because Prof. Jones revised the students' papers.

While these arguments are not intended to rule out the possibility that there are instances of VPE with telescoping quantifiers, they constitute strong evidence we cannot explain away the sticky reading as always involving telescoping quantifiers, and therefore as a sub-case of the sloppy reading. Furthermore, in the following section we argue that they constitute a problem for existing theories of VPE. For reasons of space, we will only discuss Rooth's (1992) theory, which has been especially influential in the literature.

\section{Rooth's parallelism condition}

Rooth (1992) enforces parallel binding as defined in (2) at the syntax-semantics interface via the Parallelism Condition, stated as follows. ${ }^{3}$ Here, $\llbracket \alpha \rrbracket^{g}$ is the ordinary

3 It should be noted that Rooth (1992) also postulates a syntactic identity condition, since the Parallelism Condition defined in (20) alone runs into an over-generation problem. Namely, it incorrectly predicts that implicational bridging, which is sufficient to license phonological reduction as in (ia) should also be sufficient to license VPE, as in (ib).

i. First someone told Mary about the Budget cuts, then $\operatorname{Sue}_{F} \ldots$

a. ....heard about them.

b. .*...did 〈hear about them〉.

Rooth (1992: 8)

In the following discussion we abstract away from these concerns, but the identity condition we propose, since it is semantic in nature, will ultimately needed to be supplemented by some version of 
semantic value of $\alpha$ and $\|\alpha\|^{g}$ is the focus semantic value of $\alpha$, as defined in the standard Roothian alternative semantics. ${ }^{4}$

\section{Parallelism Condition}

There must be a constituent $\mathrm{PD}_{E}$ that reflexively dominates VPE and a constituent $\mathrm{PD}_{A}$ that reflexively dominates the antecedent VP such that $\llbracket \mathrm{PD}_{A} \rrbracket^{g} \in\left\|\mathrm{PD}_{E}\right\|^{g}$ for all $g$.

This condition rules out 'mixed readings' that violate parallel binding. In order to see this, consider the following example, where the second sentence lacks the mixed reading that Prof. Jones cited Prof. Smith's paper.

* [None of the students of Prof. Smith's $\left.{ }^{y}\right]^{x}$ cited his $x$ paper. Prof. Jones did $\langle$ cite his $y$ paper $\rangle$.

Here, $\mathrm{PD}_{E}$, which we take to be the second sentence of (21), contains a free pronoun, and its focus value is as in (22). We assume that the subject 'Prof. Jones' is focused and that it denotes a generalised quantifier, and $g(y)=$ Prof. Smith.

$$
\begin{aligned}
& \|[\text { Prof. Jones }]_{F}\langle\text { cite his } y \text { paper }\rangle \|^{g} \\
& =\left\{\lambda w . Q(\lambda x . x \text { didn't cite Prof. Smith's paper in } w) \mid Q \in D_{\langle e t, t\rangle}\right\}
\end{aligned}
$$

On the other hand, $\mathrm{PD}_{A}$, which we take to be the first sentence, contains a bound variable, so its ordinary semantic value is (23).

$\llbracket\left[\text { None of the students of Prof. Smith's }{ }^{y}\right]^{x}$ cited his $x$ paper $\rrbracket^{g}$

$=\lambda w$. none of the students of Prof. Smith's cited his own paper in $w$

Clearly, $(23) \notin(22)$, so the Parallelism Condition is not satisfied and consequently VPE is not available, which is a desirable result.

The Parallelism Condition is satisfied only under parallel binding. More specifically, if the first sentence meant (24) instead, we would have $(24) \in(22)$.

$\llbracket\left[\text { None of the students of Prof. Smith's }{ }^{y}\right]^{x}$ cited his $y$ paper $\rrbracket^{g}$

$=\lambda w$. none of the students of Prof. Smith's cited Prof. Smith's paper in $w$

And indeed, this is the strict reading, which is available. Similarly, if the second sentence meant (25), we would have $(23) \in(25)$, which corresponds to the sloppy reading.

$$
\begin{aligned}
& \|[\text { Prof. Jones }]_{F}^{z}\left\langle\text { cite his }_{z} \text { paper }\right\rangle \|^{g} \\
& =\left\{\lambda w . Q(\lambda x . x \text { didn't cite } x \text { 's paper in } w) \mid Q \in D_{\langle e t, t\rangle}\right\}
\end{aligned}
$$

syntactic identity.

4 If $\alpha$ is a terminal node, then $\|\alpha\|^{g}=D_{\tau}$ where $\tau$ is the type of $\llbracket \alpha \rrbracket^{g}$. If $\alpha$ is a branching node whose children are $\beta$ and $\gamma$ such that $\llbracket \beta \rrbracket^{g} \in D_{\langle\sigma, \tau\rangle}$ and $\llbracket \gamma \rrbracket^{g} \in D_{\sigma}$, then $\|\alpha\|^{g}=$ $\left\{b(c) \mid b \in\|\beta\|^{g} \wedge c \in\|\gamma\|^{g}\right\}$. 
The sticky reading

In conclusion, given that the sticky reading has essentially the same structure as the mixed reading in (21) above, except that the pronoun in the VPE is an E-type pronoun and denotes all the students of Prof. Smith's, the Parallelism Condition undergenerates, as it incorrectly rules out such readings.

\section{Our analysis}

Instead of Rooth's (1992) Parallelism Condition, we postulate the following two conditions. Here and below, 'E-Cl' refers to the clause containing the VPE and 'A-Cl' refers to the antecedent clause.

a. Focus Condition: $\|\mathrm{E}-\mathrm{Cl}\|$ entails $\|\mathrm{A}-\mathrm{Cl}\|$.

b. Discourse Condition (Informal): $\mathrm{E}-\mathrm{Cl}$ is 'dependent' on $\mathrm{A}-\mathrm{Cl}$ in the discourse.

In what follows, we will discuss these two conditions in turn. Before moving on, however, one caveat is in order. To simplify the discussion, in this paper we will only look at VPEs in non-embedded declarative clauses (although we will see a couple of examples of VPE in questions in this section). Thus, $\mathrm{A}-\mathrm{Cl}$ and $\mathrm{E}-\mathrm{Cl}$ are assumed here to be simple declarative clauses. See Section 5 for some preliminary discussion on more complex cases.

\subsection{The focus condition}

One crucial difference between the sticky reading and other mixed readings is that under the sticky reading, the two sentences are about the same set of objects. For instance, for our initial example (3), both sentences are about the same set of papers by the same set of authors. The Focus Condition, as in (26a), is meant to capture this intuition.

The Focus Condition refers to the entailment relation between two focus values. Since both $\mathrm{A}-\mathrm{Cl}$ and $\mathrm{E}-\mathrm{Cl}$ are sentences, these focus values are sets of propositions. Thus the entailment relation is different from the canonical one that holds between two sentences or propositions. We borrow the following notion of entailment that is often used for entailment between questions (Groenendijk \& Stokhof 1984: 16).

(27) Let $Q$ and $Q^{\prime}$ be sets of propositions. $Q$ entails $Q^{\prime}$ iff every complete answer to $Q$ is a complete answer to $Q^{\prime}$.

Although the focus values of sentences are not 'questions' in the normal sense, they are the same semantic objects as question denotations, namely sets of propositions (Hamblin 1973; Karttunen 1977). Therefore, this notion of entailment can apply to them. As will become clear shortly, however, we cannot adopt the standard notion 
of complete answers here, and for the moment, we will leave this issue open, relying on the intuitive notion of complete answers instead.

To illustrate, let us revisit (3). We assume that it has the following focus structure, which we believe is the most prominent focus structure (under the sticky reading). Here SMALL CAPS indicate focus prominence, and $F$ marks the focused constituent.

(28) $\left[[\mathrm{NONE}]_{F} \text { of the authors }\right]^{x}$ proofread his $_{x}$ paper. So [the Editor $]_{F}$ did.

Then, the focus values of $\mathrm{E}-\mathrm{Cl}$ and $\mathrm{A}-\mathrm{Cl}$ are as follows. ${ }^{5}$

a. $\|[\text { the Editor }]_{F}$ did $\langle$ proofread the authors' papers $\rangle \|$ $=\left\{\lambda w . x\right.$ proofread the authors' papers in $\left.w \mid x \in D_{e}\right\}$

b. $\|\left[[\text { NONE }]_{F} \text { of the authors }\right]^{x}$ proofread his $x$ paper $\|$

$$
=\left\{\lambda w \cdot Q\left(\lambda x . \begin{array}{l}
x \text { is an } \\
\text { author in } w
\end{array}\right)\left(\lambda x . \begin{array}{l}
x \text { proofread } \\
x \text { 's paper in } w
\end{array}\right) \mid Q \in D_{\langle e t,\langle e t, t\rangle\rangle}\right\}
$$

Every complete answer to (29a) is a complete answer to (29b), or intuitively, completely knowing who proofread the authors' papers leads to the knowledge of how many of the authors proofread their own papers. Therefore, E-Cl entails $\mathrm{A}-\mathrm{Cl}$ (although not vice versa). ${ }^{6}$

Notice that whether the entailment holds crucially relies on the focus structure. Thus, we make the following prediction. When the entire quantifier DP is focused in $\mathrm{A}-\mathrm{Cl}$, the sticky reading is unavailable, because (29a) does not entail the following. Knowing who proofread the authors' paper will not help us identify who proofread their own paper.

$$
\begin{aligned}
& \|[\text { none of the AUthors }]_{F}^{x} \text { proofread his } x \text { paper } \| \\
& =\left\{\lambda w \cdot Q(\lambda x . x \text { proofread } x \text { 's paper in } w) \mid Q \in D_{\langle e t, t\rangle}\right\}
\end{aligned}
$$

This prediction is borne out: the following example lacks the sticky reading.

(31) [None of the AUthors $]_{F}$ proofread his paper. So the editor did (instead).

Under strict and sloppy readings, $\|\mathrm{E}-\mathrm{Cl}\|=\|\mathrm{A}-\mathrm{Cl}\|$ generally holds. Consequently, the Focus Condition is satisfied. Furthermore, under unavailable mixed readings, the entailment does not hold, e.g., (22) does not entail (23) (completely knowing who cited Prof. Smith's paper does not lead to a complete answer to 'Who cited his own paper').

5 Here we analyse the editor as of type $e$, unlike in (22), but this is inconsequential.

6 As remarked above, standard notions of complete answerhood (e.g., proposition $p$ is a complete answer to set $Q$ of propositions iff for each $q \in Q, p$ entails or contradicts $q$ ) do not deliver this result, as the distributive reading is assumed to underspecify which person proofread which paper (assuming that who is number-neutral and ranges over singularities as well as pluralities). In order to circumvent this problem, we need a stronger notion of complete answers, which specifies how the object plurality the authors' papers is distributed over the subject plurality who. We leave how to formalise this notion for future research. 
The sticky reading

Notice that our Focus Condition is weaker than Rooth's (1992) Parallelism Condition, and thus one needs to be careful not to overgenerate. Our second condition, which we show is independently necessary, supplements the Focus Condition and further restricts possible VPE.

\subsection{The discourse condition}

Our second condition, the Discourse Condition, is meant to capture the fact that $\mathrm{A}-\mathrm{Cl}$ and $\mathrm{E}-\mathrm{Cl}$ need to stand in a particular type of discourse relation in order for the sticky reading to be possible.

It is independently known that VPE sensitive to discourse relations between A-Cl and E-Cl (Kehler 2002; Hardt \& Romero 2004; Kehler \& Büring 2008). ${ }^{7}$ For instance, in the following example taken from Hardt \& Romero 2004, only one interpretation is possible, despite the fact that there are two potential antecedents for VPE.

(32) Agnes arrived after John ate. But Bill didn't 〈*eat / arrive after John ate $\rangle$

We observe that the sticky reading is more limited in distribution than the strict and sloppy readings. One example that illustrates this involves $\mathrm{A}-\mathrm{Cl}$ and $\mathrm{E}-\mathrm{Cl}$ answering the same question; this is an instance of two sentences standing in a PARALLEL coherence relation, in the sense of Kehler 2002. In such a situation, only strict and sloppy readings are available as shown below.

Q1: Who $^{x}$ proofread his $_{x}$ (own) paper?

A1: [None of the semAnticists] ${ }^{x}$ proofread his $_{x}$ paper.

A2: But one syntactician ${ }^{y}$ did $\left\langle\right.$ proofread his $_{y}$ paper $\rangle$.

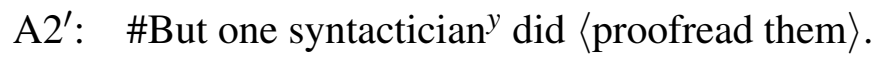

As indicated here, $\mathrm{A} 2{ }^{\prime}$ is not a possible interpretation of the VPE. We explain this observation by using the notion of congruence:

(34) A declarative sentence $S$ is congruent to question $Q$ (wrt $g$ ) iff $\llbracket Q \rrbracket^{g}=\|S\|^{g}$.

Following Roberts (2012), among others, we also assume that each discourse move (assertion or question) is required to be 'discourse-dependent' on some prior discourse move, and that an assertion always answers some prior question that is explicit or implicit in the discourse and that the assertion is congruent to. Let us state this latter constraint as follows.

7 Rooth's (1992) Parallelism Condition can be seen as a way to capture this, too. That is, regarding the focus value of $\mathrm{PD}_{E}$ as the question that $\mathrm{PD}_{E}$ is 'about', it requires there to be some $\mathrm{PD}_{A}$ that answers it. However, as Hardt \& Romero (2004) claim, this requirement itself is too weak in light of data like (32). 
(35) An assertion $A$ is discourse-dependent on a question $Q$ if $A$ is a partial answer to $Q$ and $A$ is congruent to $Q$.

Coming back to the data in (33), the discourse dependencies here are indicated by indentation. That is, all the answers are directly dependent on Q1. Then A2', although satisfying the Focus Condition, is not congruent to Q1, violating the discourse-dependency condition. Therefore it is not a licit discourse move. On the other hand, A1 and A2 are both congruent to Q1 and hence acceptable.

An important consequence of this is that sticky readings should not be found in the canonical examples of VPE of the form 'DP VP, and DP $\langle\mathrm{VP}\rangle$, too' or 'DP VP, but DP not $\langle\mathrm{VP}\rangle$ ', because in these cases, $\mathrm{A}-\mathrm{Cl}$ and $\mathrm{E}-\mathrm{Cl}$ answer the same question 'Who VP?', and (35) rules out the sticky reading.

Moreover, the present account makes a number of predictions. Firstly, due to the congruence requirement, an antecedent VP in a question should never license the sticky reading. This prediction is borne out, as shown by the following example.

Q: $\left[\right.$ Which of the authors] ${ }^{x}$ proofread his $x$ paper?

A: \#The editor did 〈proofread them〉.

On the other hand, the sticky reading is available if the e-site occurs in a question, because (35) does not apply to a question, as shown in (37).
A: [None of the authors $]^{x}$ proofread his $_{x}$ paper.
Q: Then who did 〈proofread them〉?

We have not yet defined the notion of discourse-dependency for questions. We will come back to this issue immediately below.

Now, let us re-visit the sticky reading of (3) to see how the Discourse Condition is satisfied. We analyze this example as involving implicit questions, which are indicated with parentheses in (38), broadly following Roberts's (2012) Question under Discussion theory of information structure. Or, to put it differently, when one judges this example, one accommodates these questions.

Q1: ([Which of the authors $]^{x}$ proofread his $_{x}$ paper?)

A1: [NONE of the authors $]^{x}$ proofread his $_{x}$ paper.

Q2: (So then who (else) proofread the authors' papers?)

A2: The editor did 〈proofread them〉 instead.

Notice that the two assertions answer two different questions, which are both implicit here. Notice also that we represent Q2 as dependent on Q1. This is a crucial assumption here, which relates to the notion of discourse dependency for questions.

Roberts (2012) focuses her attention to cases where one question is a more specific question of the other in the sense of contextual entailment between questions, 
The sticky reading

but to account for cases like (38), we need a more general notion of discoursedependency between questions. To this end, we propose to extend Roberts's theory by defining the notion of a Follow-up Question (FuQ) as follows (this is a tentative list of FuQs; see Kehler 2011 and Keshet 2013 for related ideas).

\section{Follow-up Question}

A question $Q$ is a Follow-up Question to another question $Q^{\prime}$ (in a discourse $D)$, if any of the following is the case:

a. Subquestion: $Q^{\prime}$ has not been completely answered in $D$ and $Q^{\prime}$ contextually entails $Q$.

b. Reason: A partial answer $p$ to $Q^{\prime}$ has been given in $D$ before $Q$ such that a partial answer to $Q$ explains $p$.

c. Consequence: A partial answer $p$ to $Q$ has been given in $D$ before $Q^{\prime}$ such that $p$ explains a partial answer to $Q^{\prime}$.

d. Concessive: A partial answer $p$ to $Q$ has been given in $D$ before $Q^{\prime}$, and $p$ makes a partial answer to $Q^{\prime}$ unlikely.

Using this, we define discourse-dependency between question as follows:

(40) Question $Q 2$ is discourse-dependent on question $Q 1$ iff $Q 2$ is a FuQ to $Q 1$.

Here is an example illustrating how this works.

(41) Q1: Who is going to NY?

A1: Each of the girls is going to NY.

Q2: Why are they all going to NY?

(reason)

A2: In order to attend SALT.

Q3: Are the boys also going to NY?

(subquestion)

A3: No, only Bill is going to NY.

Similarly, in (38), Q2 is a consequence FuQ of Q1.

Now, capitalising on the above two notions of discourse-dependency between assertions and questions in (35) and (40), we formalise the Discourse Condition on VPE as follows. Recall that in the present paper, we only analyse cases where both $\mathrm{A}-\mathrm{Cl}$ and $\mathrm{E}-\mathrm{Cl}$ are assertions.

(42) Discourse Condition (formal): The question that $\mathrm{E}-\mathrm{Cl}$ is discourse-dependent on must be discourse-dependent on the question that $\mathrm{A}-\mathrm{Cl}$ is discoursedependent on.

As one can see, this is satisfied in (38). 


\section{Conclusions and further issues}

The most important contribution of the present paper is that it identifies the sticky reading as a third reading of VPE whose antecedent VP contains a pronoun. We argued that being a type of mixed reading, the sticky reading poses a serious challenge to existing theories of VPE, which rule out all mixed readings by requiring parallel binding. To account for the sticky reading, we proposed two conditions on VPE, the Focus Condition and the Discourse Condition, repeated below in (43). In particular, the latter accounts for the requirement that the antecedent VP be found in a discourse move that stands in a particular dependency relation with the discourse move containing the VPE. We formalised this dependency relation in an extension of Roberts's (2012) QuD framework.

a. Focus Condition: $\|\mathrm{E}-\mathrm{Cl}\|$ entails $\|\mathrm{A}-\mathrm{Cl}\|$.

b. Discourse Condition: The question that $\mathrm{E}-\mathrm{Cl}$ is discourse-dependent on must be discourse-dependent on the question that $\mathrm{A}-\mathrm{Cl}$ is discoursedependent on.

Our current theory is admittedly still preliminary and leaves a number of issues open. One of them is to define the proper notion of complete answerhood and question entailment, as briefly discussed above. Another remaining issue is how to extend the present system to non-declarative sentences, e.g., (37). In the following, we mention two additional shortcomings of the present formalisation.

\subsection{Existential quantifiers}

Our theory as it stands now fails to account for sticky readings involving existential quantifiers, such as (5). Here is another such example.

(44) $\left[\mathrm{SOME}_{F} \text { of the authors }\right]^{x}$ didn't proofread their ${ }_{x}$ papers. So [the Editor $]_{F}$ did.

The second sentence has a reading where the editor proofread those papers whose authors did not proofread them. This reading is not accounted for since the focus values of the two clauses do not stand in an entailment relation. More specifically, the focus values are the following:

$$
\begin{aligned}
& \|\mathrm{E}-\mathrm{Cl}\|=\{\lambda w . \\
& \|\mathrm{A}-\mathrm{Cl}\|=\left\{\lambda w . Q_{\langle e t,\langle e t, t\rangle\rangle}\left(\lambda x, \begin{array}{l}
x \text { is an } \\
\text { author in } w
\end{array}\right)\left(\lambda x . \begin{array}{l}
x \text { didn't proofread } \\
x \text { 's paper in } w
\end{array}\right)\right\}
\end{aligned}
$$

Clearly, knowing who proofread a subset of the relevant papers does not completely answer who proofread their own paper in the general case. 
The sticky reading

This problem arises because the definite term in $\mathrm{E}-\mathrm{Cl}$ is anaphoric to the existential quantifier in $\mathrm{A}-\mathrm{Cl}$, but this anaphoric relation is ignored in the computation of the Focus Condition. We think that a promising modification of the present system would be to dynamicize the notion of entailment so as to capture the anaphoric relation, but this is not at all a trivial matter, and we need to leave it for another occasion (but see Elliott \& Sudo to appear for discussion of related issues, and an attempt at a dynamicized identity condition).

\subsection{Intra-sentential cases}

Another shortcoming of our theory is that it cannot account for sticky readings within sentences, such as the following.

(47) If [none of the authors $]^{x}$ proofread his $x$ paper, the editor will 〈proofread the authors' papers $\rangle$.

In order to account for such examples in our framework, it is necessary to extend the entire QuD framework by defining 'discourse-dependency' among embedded sentences. This might be doable, especially once the system is completely dynamicised, but this is not at all a simple issue, especially regarding how to define the meanings of connectives. For this reason, this needs to await another occasion (see Kehler 2011 and Keshet 2013 for related discussion).

\section{References}

Beck, Sigrid \& Uli Sauerland. 2000. Cumulation is needed: A reply to Winter (2000). Natural Language Semantics 8(4). 349-371.

Büring, Daniel. 2005. Binding Theory. Cambridge: Cambridge University Press.

Dalrymple, Mary, Stuart M. Shieber \& Fernando C. N. Pereira. 1991. Ellipsis and higher-order unification. Linguistics and Philosophy 14(4). 399-452.

Elliott, Patrick D. \& Yasu Sudo. to appear. E-type readings of quantifiers under ellipsis. In Proceedings of Sinn und Bedeutung 20, .

Fiengo, Robert \& Robert May. 1994. Indices and Identity, vol. 24 Linguistic Inquiry Monographs. MIT press.

Groenendijk, Jeroen \& Martin Stokhof. 1984. Studies on the semantics of questions and the pragmatics of answers: University of Amsterdam dissertation.

Hamblin, Charles Leonard. 1973. Questions in Montague English. Foundations of Language 10. 41-53.

Hardt, Daniel \& Maribel Romero. 2004. Ellipsis and the structure of discourse. Journal of Semantics 21. 375-414. 
Heim, Irene \& Angelika Kratzer. 1998. Semantics in Generative Grammar. Oxford: Blackwell.

Karttunen, Lauri. 1977. Syntax and semantics of questions. Linguistics and Philosophy 1. 3-44.

Kehler, Andrew. 2002. Coherence, Reference, and the Theory of Grammar. Stanford: CSLI.

Kehler, Andrew. 2011. Cohesion and coherence. In Klaus von Heusinger, Claudia Maiernborn \& Paul Portner (eds.), Semantics: An International Handbook of Natural Language Meaning, vol. 2, 1963-1987. de Gruyter Mouton.

Kehler, Andrew \& Daniel Büring. 2008. Be bound or be disjoint. In Anisa Schardl, Martin Walkow \& Muhammad Abdurrahman (eds.), Proceedings of NELS 38, 487-501. Amherst, MA: GSLA.

Keshet, Ezra. 2008. Telescoping and scope economy. In Charles B. Chang \& Hannah J. Haynie (eds.), Proceedings of WCCFL 26, 324-331. Cascadilla.

Keshet, Ezra. 2013. Sloppy identity unbound. In Todd Snider (ed.), Semantics and Linguistic Theory (SALT) 23, 412-431. LSA.

Lappin, Shalom. 1992. The syntactic basis of ellipsis resolution. In Steve Berman \& Arild Hestvik (eds.), Proceedings of the Stuttgart Workshop on Ellipsis, Arbeitspapiere des Sonderforschungsbereich 340, Bericht Nr. 29-1992.

Poesio, Massimo \& Alessandro Zucchi. 1992. On telescoping. In Chris Barker \& David Dowty (eds.), Semantics and Linguistic Theory (SALT) 2, 347-367. LSA.

Roberts, Craige. 2012. Information structure: Towards an integrated formal theory of pragmatics. Semantics and Pragmatics 5. 1-69.

Rooth, Matts. 1992. Ellipsis redundancy and reduction redundancy. In Steve Berman \& Arild Hestvik (eds.), Proceedings of the Stuttgart Ellipsis Workshop, 1-26.

Sag, Ivan A. 1976. Deletion and logical form: Massachusetts Institute of Technology dissertation. 
The sticky reading

Patrick D. Elliott

University College London

Chandler House

2 Wakefield St

London WC1N 1PF

UK

p.elliott@ucl.ac.uk

Yasutada Sudo

University College London

Chandler House

2 Wakefield St

London WC1N 1PF

UK

y.sudo@ucl.ac.uk
Andreea C. Nicolae

Zentrum für Allgemeine

Sprachwissenschaft

Schützenstr. 18

D-10117 Berlin

Germany

andreea.nicolae@gmail.com 